\title{
Transitional Use
}

National Cancer Institute

\section{Source}

National Cancer Institute. Transitional Use. NCI Thesaurus. Code C118646.

Employing something to be used prior to a more permanent solution. 